\title{
Endoscopic Total Corpus Callosotomy: Cadaveric Demonstration of a New Approach
}

\author{
Biji Bahuleyan $^{\text {a }}$ Timothy W. Vogel $^{\mathrm{b}}$ Shenandoah Robinson ${ }^{\mathrm{b}}$ Alan R. Cohen ${ }^{\mathrm{b}}$ \\ a Division of Pediatric Neurosurgery, Rainbow Babies and Children's Hospital, The Neurological Institute, \\ Case Western Reserve University School of Medicine, Cleveland, Ohio, and ${ }^{\text {H}}$ Harvard Medical School, Boston, Mass., USA
}

Key Words

Callosotomy $\cdot$ Endoscopy $\cdot$ Epilepsy

\begin{abstract}
Background: Callosotomy is a surgical option for treatment of a small number of patients with intractable seizures who are not candidates for surgical resection. The procedure is done conventionally with the aid of a microscope. In this article, we describe a new technique of endoscopic callosotomy through a parasagittal burr hole in a cadaveric model. Methods: We utilized this technique with a single frontal burr hole. Callosotomies were performed on 4 silicon-injected cadaver heads in our minimally invasive neurosurgical laboratory. Results: We were able to successfully perform total callosotomies in all cadaveric specimens without injury to the adjacent neurovascular structures. The advantages of our technique over microsurgery are the minimally invasive nature of the exposure and the improved visualization of the vascular structures near the rostrum and genu of the corpus callosum. A potential disadvantage we anticipate is the difficulty controlling bleeding in the event of vascular injury. Conclusion: We believe that this minimally invasive approach could have potential clinical applications.
\end{abstract}

Copyright $\odot 2012$ S. Karger AG, Base
(C) 2012 S. Karger AG, Basel $1016-2291 / 11 / 0476-0455 \$ 38.00 / 0$

Fax +41613061234 E-Mail karger@karger.ch www.karger.com

\section{Introduction}

The corpus callosum is a set of commissural fiber that transmits impulses from one hemisphere to the other. In patients with seizures, this major white matter bundle serves to propagate epileptic discharges between cerebral hemispheres. Division of the corpus callosum, corpus callosotomy, has been shown to significantly reduce seizures in a subgroup of patients with intractable epilepsy [1]. The mechanism of seizure reduction is interruption of the spread of epileptic impulses from one hemisphere to the other.

Corpus callosotomy is traditionally done microsurgically through a parasagittal craniotomy on the nondominant side. There are two prior reports of endoscopic callosotomy $[2,3]$. A cadaveric study by Guerrero and Cohen [2] described the usefulness of the endoscope in aiding microsurgical callosotomy. Tubbs et al. [3] demonstrated the technique of anterior callosotomy through a burr hole just above the nasion.

In this technical note, we describe the relevant microsurgical anatomy and endoscopic techniques for total callosotomy through a single frontal burr hole. In all four specimens, we introduced the endoscope through a parietal parasagittal burr hole to study the completeness of the procedure. 


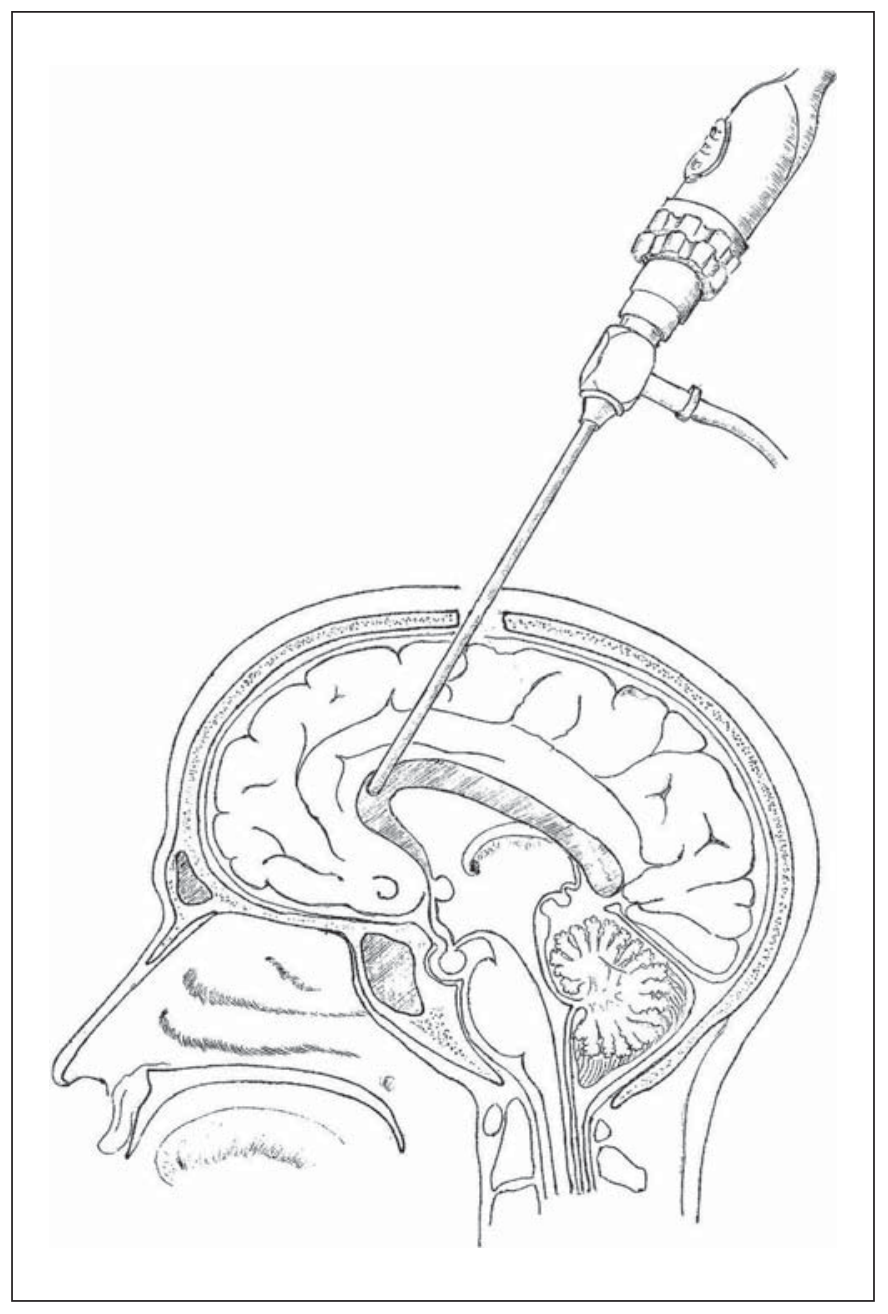

Fig. 1. Schematic diagram showing endoscopic visualization of anterior corpus callosum through a frontal parasagittal burr hole.

\section{Materials and Methods}

We used 4 silicon-injected cadaver heads for the procedure in our minimally invasive neurosurgical laboratory. The cadaver head was positioned supine in a neutral position with slight flexion. Right frontal and parietal parasagittal burr holes were made with a power perforator. The frontal burr hole was made just anterior to the coronal suture and the parietal burr hole was made $4 \mathrm{~cm}$ posterior to the coronal suture. The medial border of each burr hole reached the lateral border of the superior sagittal sinus. The burr holes were undercut anteriorly and posteriorly sufficiently enough to enable introduction and comfortable angulation of the endoscope to visualize the genu (fig. 1) and splenium (fig. 2) of the corpus callosum. The dura was opened in a cruciate fashion and a rigid endoscope (Aesculap, Melsungen, Germany) was inserted parasagittally. The obturator was removed and a 2.7-mm-diameter zero-degree straightforward rod lens was inserted. The image was transmitted to a high-definition video

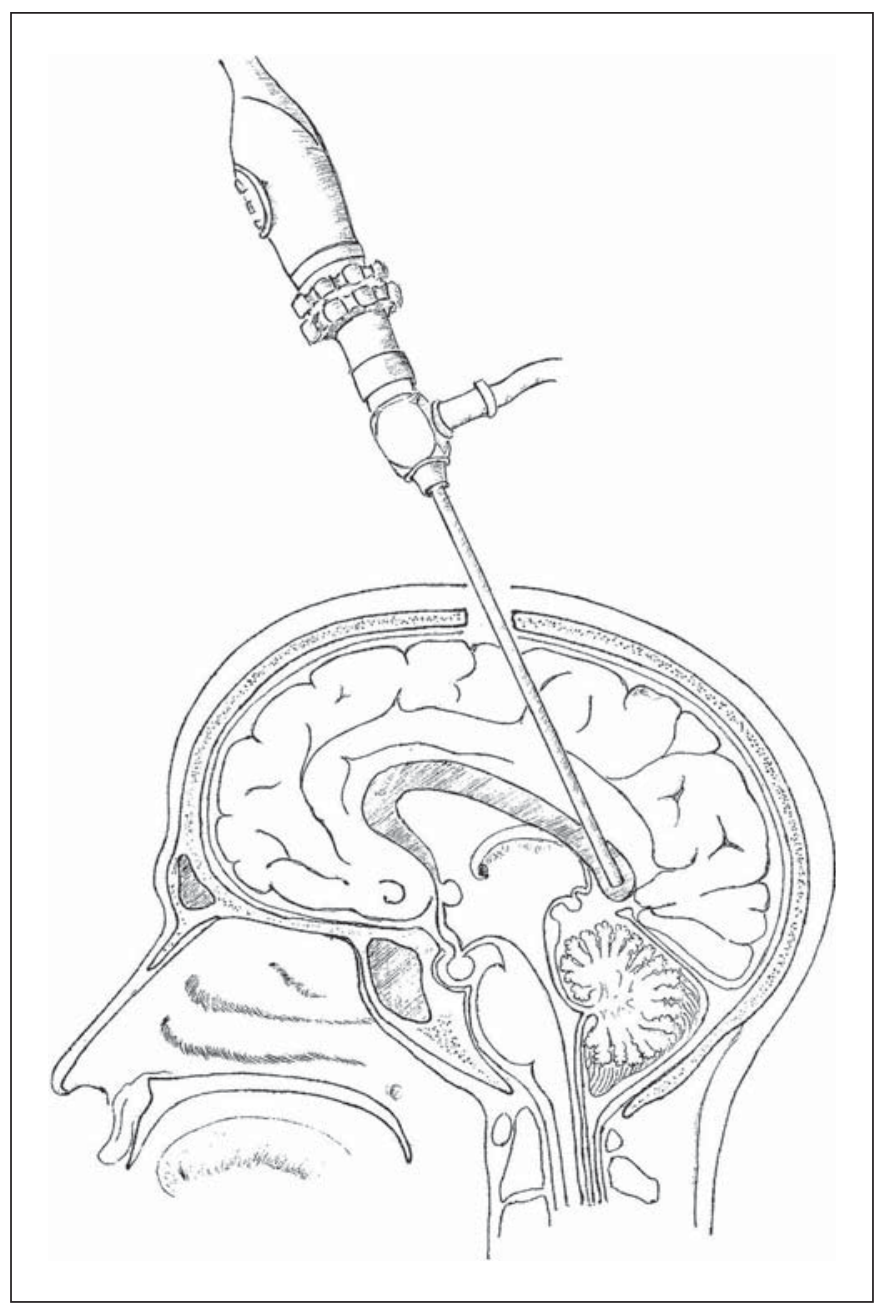

Fig. 2. Schematic diagram showing endoscopic visualization of posterior corpus callosum through a frontal parasagittal burr hole.

monitor using a microchip camera and photographs were taken. White matter disconnections were created using a blunt probe and endoscope scissors inserted through a working channel in the endoscope sheath. Total callosotomy was performed in all $4 \mathrm{ca}-$ daver heads through the frontal burr hole. Endoscopic view through the parietal burr hole was done to assess the completion of the procedure.

\section{Results}

\section{Predissection Endoscopic Anatomy}

The endoscope was advanced vertically through the frontal burr hole along the right side of the falx cerebri toward the superior surface of the corpus callosum. There were numerous arachnoid adhesions but few venous 
Fig. 3. a Endoscopic view of the anterior aspect of the corpus callosum through a right parasagittal frontal burr hole showing the genu (G), body (B), falx cerebri (star), pericallosal artery (arrow) and callosomarginal artery (arrowhead). b Endoscopic view of the posterior aspect of the corpus callosum through a right parasagittal frontal burr hole showing the posterior limit of the splenium (S) and the inferior curve of the falx (star) behind the splenium.
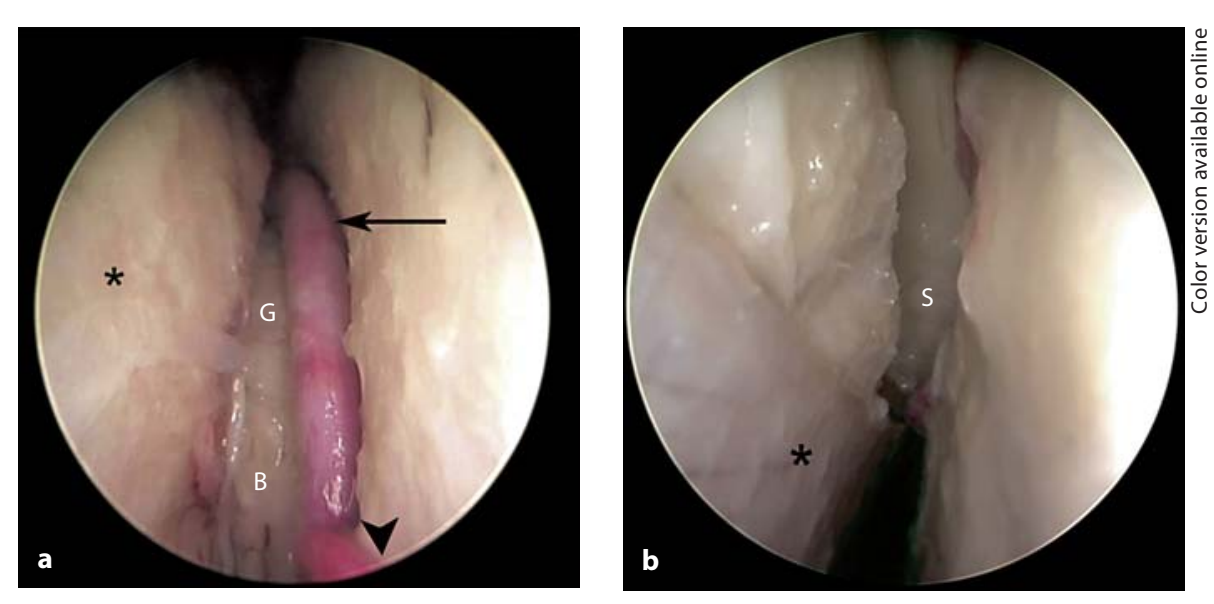

channels between the medial surface of the right hemisphere and the falx. The number of adhesions increased at the inferior border of the falx. These adhesions were released with endoscopic microscissors and gentle retraction of the right hemisphere away from the falx. During dissection, intermittent gentle retraction of the medial surface of the hemisphere to the right side with the endoscope enabled good exposure of the area of dissection.

The callosum was seen a few millimeters inferior to the inferior margin of the falx in all 4 hemispheres. The superior surface of the callosum appeared as a glistening white structure with small callosal arteries supplying it. Anteriorly, the genu of the callosum was identified by its sharp bend inferiorly and the right pericallosal artery making a wide arc around the genu to lie on its superior surface (fig. 3a). Posteriorly, the superior surface of the corpus callosum was appreciated extending to the region of the splenium. The posterior extent of the callosum was confirmed anatomically by following the inferior border of the falx cerebri as it curved inferiorly behind the splenium (fig. 3b).

In all 4 cadaver heads, the right pericallosal artery was seen curving around the genu of the corpus callosum within the callosal sulcus (fig. 3a). The right callosomarginal artery was seen arising from the pericallosal artery posteriosuperior to the genu. The callosomarginal artery curved superiorly initially and then posteriorly to lie within the cingulate sulcus. On tracing the right pericallosal artery posteriorly, it was seen extending posteriorly up to the splenium in 3 hemispheres. In 1 hemisphere, the pericallosal artery ended near the body of corpus callosum. In this hemisphere, the terminal branches of the callosomarginal artery curved inferiorly from the cingulate sulcus to supply the posterior aspect of the body and the splenium. In 1 hemisphere, a small branch from the distal right pericallosal artery was seen crossing the midline to supply the left hemisphere.

We did not visualize any parasagittal draining veins at the site of the frontal and parietal burr holes.

\section{Dissection of the Genu, Rostrum and Anterior Aspect of the Body of Corpus Callosum}

We used multiple small cotton balls in the interhemispheric fissure away from the trajectory of dissection to aid retraction of the hemisphere from the falx. The pial layer over the superior surface of the corpus callosum was sectioned and dissection was advanced inferiorly in a vertical plane. The septum pellucidum was seen in all the hemispheres. In 2 heads, callosotomy was in the midline and hence the septum pellucidum was split into a right and left half (fig. 4a). We were able to differentiate the junction between the inferior border of the corpus callosum and the beginning of the superior extent of the septum pellucidum by the change in color of the tissue: the corpus callosum appearing slightly red and the septum pellucidum appearing slightly blue. Identification of this demarcation is important in assessing completeness of the vertical extent of the procedure. In the other two cadavers, our plane of dissection was slightly off midline and hence we entered the right lateral ventricle after breaching its ependymal surface at the inferior surface of the callosum. In these 2 hemispheres we could identify the septum pellucidum on the left side of our dissection.

In the specimen in which a branch of the pericallosal artery was supplying the opposite hemisphere, the callosal dissection was done on either side of the vessel and then the callosal tissue was undercut beneath the vessel with an endoscopic forceps. 
Fig. 4. a Endoscopic view of the anterior aspect of the corpus callosum after callosotomy through right parasagittal frontal burr hole, showing the split genu $(\mathrm{G})$, the septum pellucidum (S), the pericallosal artery (arrow) and the callosomarginal artery (arrowhead). b Endoscopic view of the posterior aspect of the corpus callosum after callosotomy through a right parasagittal frontal burr hole, showing the split posterior aspect of the body of the corpus callosum (B), the spilt splenium (S) and the internal cerebral veins (arrows).
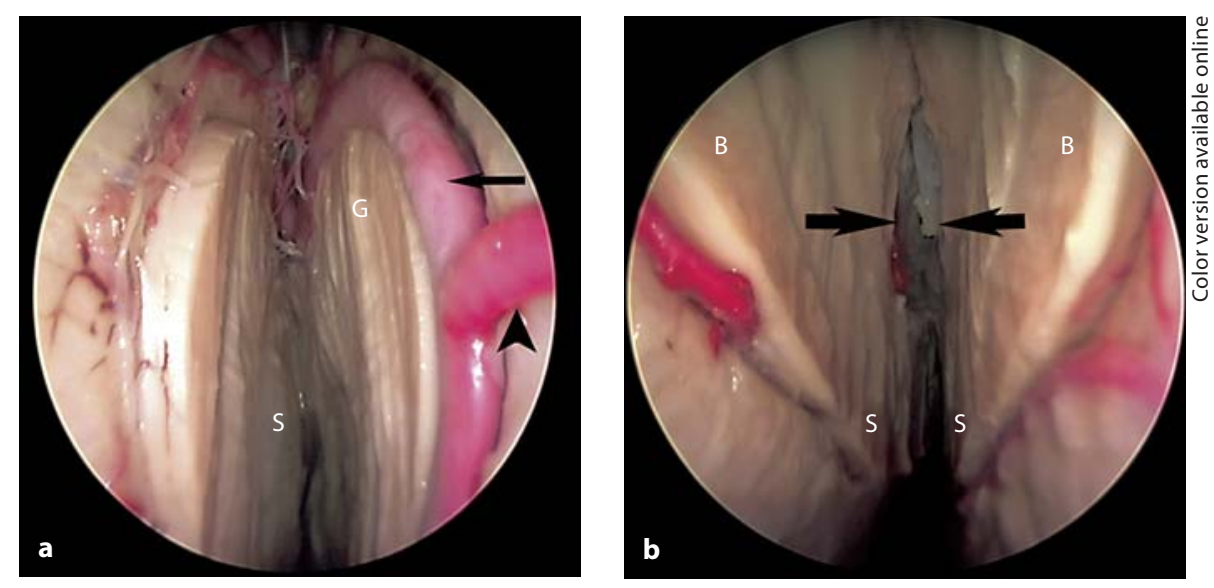

The anterior extent of our dissection was till the genu and rostrum of the corpus callosum. The rostrum could be identified as the dissection of the genu advanced inferiorly and its thickness gradually tapered off. During dissection of the rostrum we also identified small perforators from the proximal anterior cerebral artery. The dissection was advanced in a vertical plane as far posteriorly on the body of the corpus callosum.

\section{Dissection of the Posterior Aspect of the Body and \\ Splenium of the Corpus Callosum}

As the dissection advanced posteriorly, the thickness of the corpus callosum increased and the vertical height of the septum gradually decreased. The septum tapered off and disappeared in front of the splenium of corpus callosum. It was difficult for us to differentiate the hippocampal commissure and the septum pellucidum at this point. On splitting the septum pellucidum completely, we identified a pair of internal cerebral veins lying in the velum interpositum (fig. 4b). The callosotomy was advanced posteriorly and its completeness was confirmed by identifying the posterior and inferior margins of the splenium.

Comparison of the Endoscopic Anatomy when Viewed through Frontal and Parietal Burr Holes

After completing total callosotomy through a frontal burr hole, we confirmed the callosotomy through a posterior bur hole $4 \mathrm{~cm}$ posterior to the coronal suture. We introduced the endoscope through the parietal burr hole to visualize the area of dissection. Endoscopic visualization through the parietal burr hole confirmed that a corpus callosotomy can be performed through a single anterior burr hole.
We were able to visualize the region of the rostrum and the splenium through both the frontal and parietal burr holes. However, visualization of the proximal anterior cerebral artery and the rostrum was better through the frontal burr hole while the visualization of the paired internal cerebral vein and the splenium was better through the parietal burr hole. The distance to the rostrum and the lower border of the splenium was measured from the frontal and parietal burr holes. The average distance to the rostrum and splenium from the frontal burr hole was 6.3 and $9.1 \mathrm{~cm}$, respectively. The distance to the rostrum and splenium from the parietal burr hole was 8.1 and $7.2 \mathrm{~cm}$, respectively. Visualization of these structures is easier if they are closer to the burr hole.

\section{Duration of the Procedure}

The approximate time taken for completion of the procedure was about $2 \mathrm{~h}$ in all 4 cadaveric heads studied.

\section{Discussion}

Van Wagnen and Harren [4] first described corpus callosotomy in 1940 after observing improvement in seizures following a stroke involving the corpus callosum. Since then, corpus callosotomy has been included in the surgical armamentarium for a small subgroup of patients with intractable seizures in whom resective procedures are not feasible [5]. Corpus callosotomy can either be total or partial [6]. Partial callosotomy can involve either an anterior or posterior disconnection. The extent of disconnection of an anterior callosotomy varies according to the choice of the surgeon. Some surgeons prefer disconnection of the anterior two thirds, while others prefer 
removal of the anterior three quarters. Studies have shown that seizure outcome is better following total callosotomy [1]. Even though seizure outcome following partial callosotomy is less favorable compared to total callosotomy, partial callosotomy has the advantage of less memory impairment and a lower incidence of a disconnection syndrome. These advantages make partial callosotomy the first treatment option in many centers. If seizures do not improve following partial callosotomy, a complete posterior callosotomy can be done $[5,6]$. Recently, with the introduction of vagal nerve stimulation, the number of corpus callosotomies has decreased considerably. Still, the procedure remains as a treatment for those patients in whom seizures are not well controlled with vagal nerve stimulation [7].

Today, the technique of partial and total callosotomy is done with the aid of an operating microscope. Guerrero and Cohen [2] described the usefulness of an endoscope in aiding microsurgical callosotomy. They found that the endoscope enabled better visualization of the most anterior and posterior aspects of the corpus callosum, which would have been more difficult with a microscope alone. The minimally invasive technique of callosotomy described by Tubbs et al. [3] in a cadaveric model involves sectioning of the anterior callosum. They performed the procedure through a paramedian hole placed $2 \mathrm{~cm}$ above the nasion. They found no vascular injury in any of the cadaveric specimens they studied. This approach, however, may have limitations in a clinical setting given the altered cosmetic appearance on the patient's forehead.

In this cadaveric study, our aim was to discuss the relevant endoscopic anatomy and techniques involved in performing total callosotomy through a single anterior or posterior parasagittal bur hole. We also discuss the potential advantages and disadvantages of our technique over the conventional microsurgical technique.

An important surgical consideration in our technique for placement of burr holes is the location of the parasagittal draining veins. We did not encounter parasagittal draining veins beneath the frontal and parietal burr holes in any of the heads we studied. While descending along the falx towards the callosum, the cingulate gyri can occasionally be adherent to one another. This can cause the surgeon to misinterpret the cingluate gyrus as the callosum. The presence of gray matter and increased vascularity of the cingulate gyrus can often help differentiate it from the white, glistening and relatively avascular corpus callosum. We did not find adhesions between cingulate gyri in any of the cadavers we studied.

Endoscopic Total Corpus Callosotomy
The major blood vessel of concern during corpus callosotomy is the distal anterior cerebral artery called the pericallosal artery by some authors [8]. Knowledge of anatomic variations of this vessel is important for microsurgical and endoscopic procedures in the interhemispheric fissure. Anatomic variations of this vessel are described by Türe et al [8] and Perlmutter and Rhoton [9].

\section{Advantages of Endoscopic Total Callosotomy over Microsurgical Total Callosotomy}

The major advantage of our technique is that it is minimally invasive, requiring only a single burr hole and thus eliminating the need for a craniotomy. Since our procedure does not require the use of retractors, there is less chance of injury to the cortex. Minimal retraction of the hemisphere in our technique also reduces the chance of rupture of the parasagittal veins. The endoscope can provide better visualization of the region of the genu and splenium of the corpus callosum by helping the surgeon look around corners to avoid vascular injury.

\section{Disadvantages of Endoscopic Total Callosotomy over Microsurgical Total Callosotomy}

The major disadvantage of endoscopic total callosotomy is that it can be difficult to control bleeding in the event of a vascular injury.

\section{Clinical Translation}

Planning the position of the burr holes can be aided with preoperative MR venograms to locate the position of the parasagittal draining veins. The head should be positioned supine. Because the callosum is a midline structure, its displacement is minimal during the procedure. Neuronavigation can serve as an excellent tool in aiding the dissection, especially while working at the genu and splenium. The burr hole should be placed on the right side except in cases where the pathology has greatly damaged the left hemisphere. The use of bipolar cautery and endoscopic scissors can aid in the sectioning of bridging veins between the falx and the medial surface of the frontal and parietal lobes.

We found that both the anterior and posterior burr holes yielded visualization of the anterior and posterior margins of the corpus callosum. It is possible, therefore, that the total corpus callosotomy could be performed through a single anterior or posterior approach. The dural opening can be closed primarily with sutures. And, if the size of the burr hole is large, the bony defect can be closed with appropriate prosthesis.

Pediatr Neurosurg 2011;47:455-460 
We have shown the efficacy of minimally endoscopic total callosotomy in a cadaveric model. The technique is easy and may benefit patients with intractable seizures who are candidates for corpus callosotomy. The location of the parasagittal burr hole can be modified depending on the position of the parasagittal bridging veins. An anteriorly placed burr hole helps better visualization of the genu, rostrum and the anterior cerebral artery while a posteriorly placed burr hole aids visualization of the region of the splenium. We believe that this technique could be applied in a clinical setting.

\section{Acknowledgements}

We thank Dr. Ajith Rajappan Nair, Assoc. Prof. of Neurosurgery and Dr. LS Jyothish, Assist. Prof. of Neurosurgery, Department of Neurosurgery, Medical College, Alappuzha, Kerala, India, for the schematic diagrams.

\section{References}

1 Rathore C, Abraham M, Rao RM, George A, Sankara Sarma P, Radhakrishnan K: Outcome after corpus callosotomy in children with injurious drop attacks and severe mental retardation. Brain Dev 2007;29:577-585.

$\checkmark 2$ Guerrero MH, Cohen AR: Endoscope-assisted microsurgery of the corpus callosum. Minim Invasive Neurosurg 2003;46:54-56.

-3 Tubbs RS, Smyth MD, Salter G, Doughty K, Blount JP: Eyebrow incision with supraorbital trephination for endoscopic corpus callosotomy: a feasibility study. Childs Nerv Syst 2004;20:188-191.
-4 van Wagenen WP, Herren RY: Surgical division of the commissural pathways in the corpus callosum. Relation to spread of an epileptic attack. Arch Neurol Psychiatry 1940; 44:740-759.

5 Tanriverdi T, Olivier A, Poulin N, Andermann F, Dubeau F: Long-term seizure outcome after corpus callosotomy: a retrospective analysis of 95 patients. J Neurosurg 2009; 110:332-342.

6 Jalilian L, Limbrick DD, Steger-May K, Johnston J, Powers AK, Smyth MD: Complete versus anterior two-thirds corpus callosotomy in children: analysis of outcome. J Neurosurg Pediatr 2010;6:257-266.
7 Roberts DW, Siegel AM: Section of the corpus callosum for epilepsy; in Schmidek $\mathrm{HH}$, Roberts DW (eds): Schmidek and Sweet Operative Neurosurgical Techniques: Indications, Methods and Results. Philadelphia, Saunders, 2006, pp 1413-1421.

$>8$ Türe U, Yaşargil MG, Krisht AF: The arteries of the corpus callosum: a microsurgical anatomic study. Neurosurgery 1996;39:10751084

$\checkmark 9$ Perlmutter D, Rhoton AL Jr: Microsurgical anatomy of the distal anterior cerebral artery. J Neurosurg 1978;49:204-228. 